\title{
Effect of exercise and high-fat diet on plasma adiponectin and nesfatin levels in mice
}

\author{
HUHE CHAOLU $^{1}$, AKIHIRO ASAKAWA ${ }^{1}$, MIHARU USHIKAI ${ }^{1}$, YING-XIAO LI ${ }^{1}$, \\ KAI-CHUN CHENG ${ }^{1}$, JIANG-BO LI ${ }^{1}$, TAKAHIRO ZOSHIKI ${ }^{1}$, MUTSUMI TERASHI ${ }^{1}$, \\ CHIE TANAKA $^{3}$, KAORI ATSUCHI ${ }^{1}$, TAKEO SAKOGUCHI ${ }^{1}$, MINGLUN TSAI ${ }^{1}$, \\ HARUKA AMITANI $^{1}$, MASAHISA HORIUCHI ${ }^{2}$, TORU TAKEUCHI ${ }^{2}$ and AKIO INUI ${ }^{1}$
}

${ }^{1}$ Department of Psychosomatic Internal Medicine; Divisions of ${ }^{2}$ Environmental Medicine, and ${ }^{3}$ Oral and Maxillofacial Surgery, Kagoshima University Graduate School of Medical and Dental Sciences, Kagoshima 890-8520, Japan

Received December 15, 2010; Accepted January 18, 2011

DOI: 10.3892/etm.2011.199

\begin{abstract}
Lifestyle-related diseases are associated with overeating and lack of exercise. The purpose of this study was to investigate the effect of exercise and high-fat diet on plasma adiponectin and nesfatin levels. Mice were housed for 4 weeks in 4 groups, which included the non-exercise and normal diet (SN), exercise and normal diet (EN), non-exercise and high-fat diet (SF) and the exercise and high-fat diet (EF) group. The mice in the exercise groups were housed in cages with a running wheel and were subjected to voluntary exercise. The food intake (Kcal) of the mice in the exercise groups increased compared to that of the mice in the non-exercise groups $(\mathrm{P}<0.01)$. Body weight and visceral fat decreased in the mice in the EF group compared to the mice in the SF group $(\mathrm{P}<0.01$ and $\mathrm{P}<0.05)$. The temperature of the mice in the EF group increased compared to that of the mice in the SN group $(\mathrm{P}<0.05)$. Blood glucose, insulin $(\mathrm{P}<0.01)$, cholesterol $(\mathrm{P}<0.01)$ and triglyceride concentrations $(\mathrm{P}<0.01)$ increased in the SF group compared to the normal diet groups. Furthermore, plasma insulin and cholesterol concentrations increased in the SF group compared to the exercise groups $(\mathrm{P}<0.01)$. Plasma adiponectin and nesfatin-1 levels in the SF group decreased compared to the $\mathrm{SN}$ group $(\mathrm{P}<0.05)$. Exercise under a high-fat diet antagonized the significant decrease in the nesfatin-1 level. Exercise together with a high-fat diet affected the plasma levels of adiponectin and nesfatin. It is therefore suggested that exercise together with a high-fat diet can affect various diseases via adiponectin and nesfatin.
\end{abstract}

Correspondence to: Dr Akihiro Asakawa, Department of Psychosomatic Internal Medicine, Kagoshima University Graduate School of Medical and Dental Sciences, 8-35-1 Sakuragaoka, Kagoshima 890-8520, Japan

E-mail: asakawa@m2.kufm.kagoshima-u.ac.jp

Key words: exercise, adiponectin, high-fat diet, nesfatin-1, mouse

\section{Introduction}

Obesity, diabetes and hyperlipidemia are lifestyle-related diseases that are receiving attention throughout the world. It is known that the development and progression of lifestylerelated diseases are closely associated with overeating, lack of exercise and heredity $(1,2)$. The lack of exercise not only increases fat that stores surplus energy, but also decreases the function of bones and muscles.

Adiponectin is a protein hormone, that is produced and secreted exclusively by adipocytes, regulates the metabolism of lipids and glucose, and exhibits anti-inflammatory properties. It has been reported to play a role in the inhibition of the development of cardiovascular diseases, type 2 diabetes and atherosclerosis, and its levels have been shown to be suppressed in insulin-resistant and obese animals and humans (3-5). Adiponectin levels have been reported to rise in response to weight loss and glitazone therapy (6-8).

Nesfatin- 1 is a recently discovered anorexigenic peptide encoded as the precursor protein nucleobindin-2 (9). Nesfatin-1 is distributed in peripheral tissues, such as adipose tissue, and also exists in the blood (9-11). A recent study reported that nesfatin-1 immunoreactivity is also detected in the rat gastric oxyntic mucosa (12). The intraperitoneal administration of nesfatin-1 inhibits food intake and thereby reduces body weight (13). Notably, nesfatin-1 also inhibits food intake in Zucker fatty rats whose leptin receptor is mutated, indicating that the anorexigenic effect of nesfatin-1 is leptin-independent (9). Nesfatin-1 is relatively stable in the circulation and it has a moderate influx from the blood to the brain compared to other peptides of similar size $(8,9,14)$. Nesfatin-1 is also present in the hypothalamus, including the paraventricular nucleus, supraoptic nucleus, arcuate nucleus, the lateral hypothalamic area and the nucleus tractus solitarius in the brain stem $(9,10,15,16)$. Nesfatin-1 suppresses eating behavior not only by peripheral administration, but also by central administration (13), and is thus expected to improve glucose metabolism in conditions such as impaired glucose tolerance, through the suppression of food intake and body weight gain. In this study, the effect of exercise and 

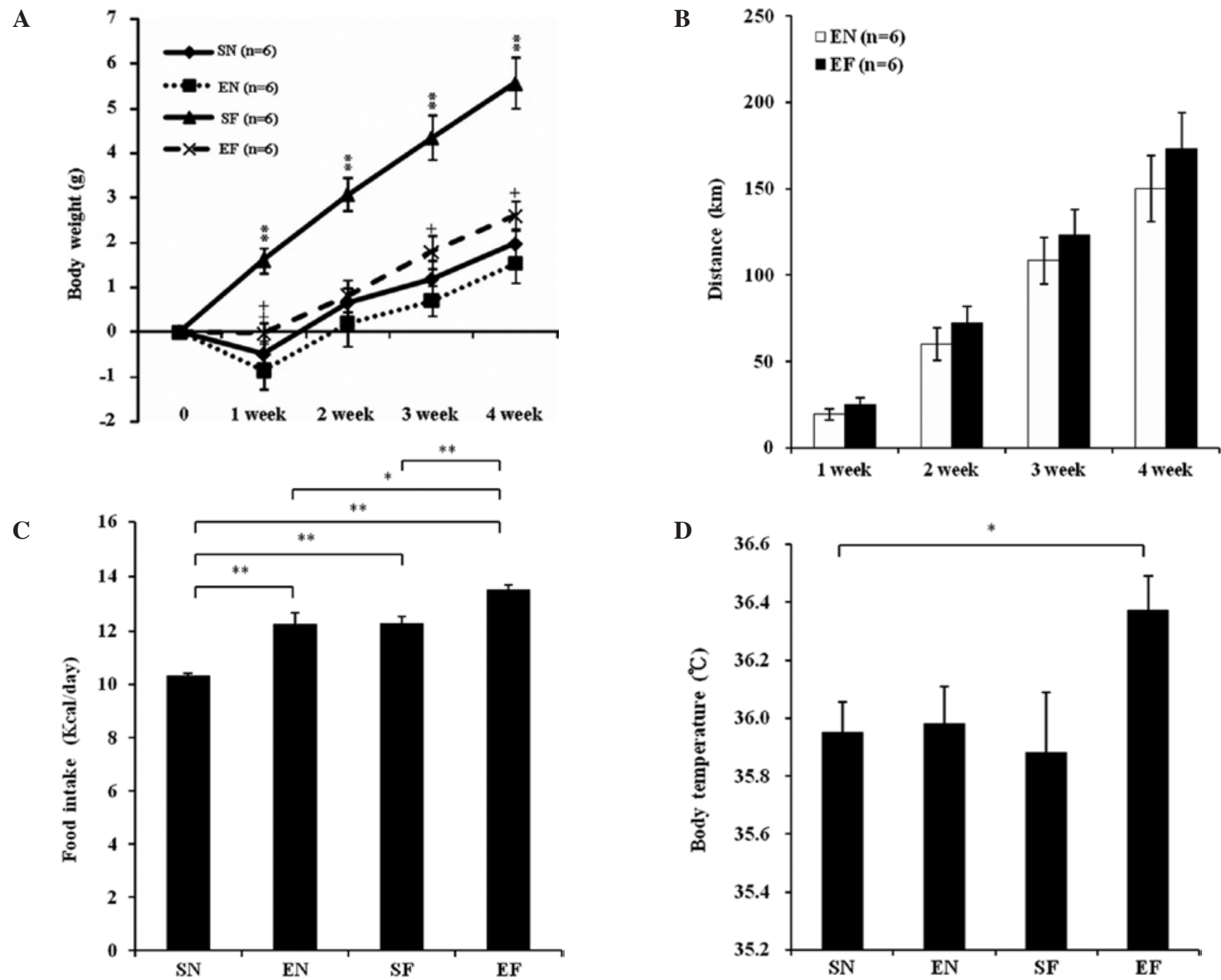

Figure 1. Effect of exercise and high-fat diet in C57BL/6J mice on (A) body weight. ${ }^{+} \mathrm{P}<0.05$ and ${ }^{++} \mathrm{P}<0.01$, difference EF vs. EN. ${ }^{* *} \mathrm{P}<0.01$ compared to other groups, (B) wheel running distance, (C) food intake. "P $<0.05$ and ${ }^{* *} \mathrm{P}<0.01$, (D) body temperature. ${ }^{*} \mathrm{P}<0.05$, difference EF vs. SN. SN, normal diet in nonexercise groups; EN, normal diet in exercise groups; SF, high-fat diet in non-exercise groups; $\mathrm{EF}$, high-fat diet in exercise groups.

high-fat diet on the levels of adiponectin and nesfatin-1 was investigated.

\section{Materials and methods}

Laboratory animals and breeding environment. Male C57BL/6J mice (21.06 \pm 0.60 g, 7-week-old; CLEA Japan, Inc., Tokyo, Japan) were used in this study. The mice were maintained in breeding rooms at a temperature of $22 \pm 1^{\circ} \mathrm{C}$ and a humidity of $55 \pm 11 \%$ under 12-h light/dark cycles, wherein the light cycle began at 7:00 am daily. The mice were individually housed. All experiments were approved by the animal experimental ethics committee of our university.

Experimental procedures. Twenty-four male C57BL/6J mice were divided into 4 groups and housed for 4 weeks. Six mice were included in each of the 4 groups: the non-exercise and normal diet (SN), exercise and normal diet (EN), non-exercise and high-fat diet (SF) and the exercise and high-fat diet (EF) group. All cages were equipped with a running wheel. The running wheels were locked for the non-exercise groups, while the exercise groups were subjected to voluntary wheel running.
The C57BL/6J mice of the normal diet group were fed daily with a normal diet (CE-2 diet containing 11\% kcal \%fat, 59\% kcal \%carbohydrate and 30\% kcal \%protein; CLEA Japan, Inc.). The C57BL/6J mice of the high-fat diet group were fed with a high-fat diet (D12492 diet containing 60\% kcal \%fat, $20 \%$ kcal \%carbohydrate and $20 \%$ kcal \%protein; Research Diets, Inc., NJ, USA). Water was available ad libitum. Motor activity, food intake and body weight were measured at the same time each day. Running distances were calculated as the number of rotations of the running wheel $x$ the circumference of the wheel $(0.7 \mathrm{~m})$. Four weeks later, the mice were deprived of food for $6 \mathrm{~h}$ for tissue sampling. Rectal temperature was determined using a digital thermometer (Technol Seven Co. Ltd., Yokohama, Japan) in a room maintained at $22 \pm 0.5^{\circ} \mathrm{C}$. A lubricated thermocouple was inserted $1.5 \mathrm{~cm}$ into the rectum of conscious mice. Blood samples were obtained from the orbital sinus under diethyl ether anesthesia. The mice were sacrificed by cervical dislocation. Visceral fat was removed and weighed. Plasma glucose was measured by the glucose oxidase method. Plasma insulin was measured by an enzyme-linked immunosorbent assay (ELISA) kit (Morinaga, Co., Tokyo, Japan). Plasma cholesterol and triglyceride 
A

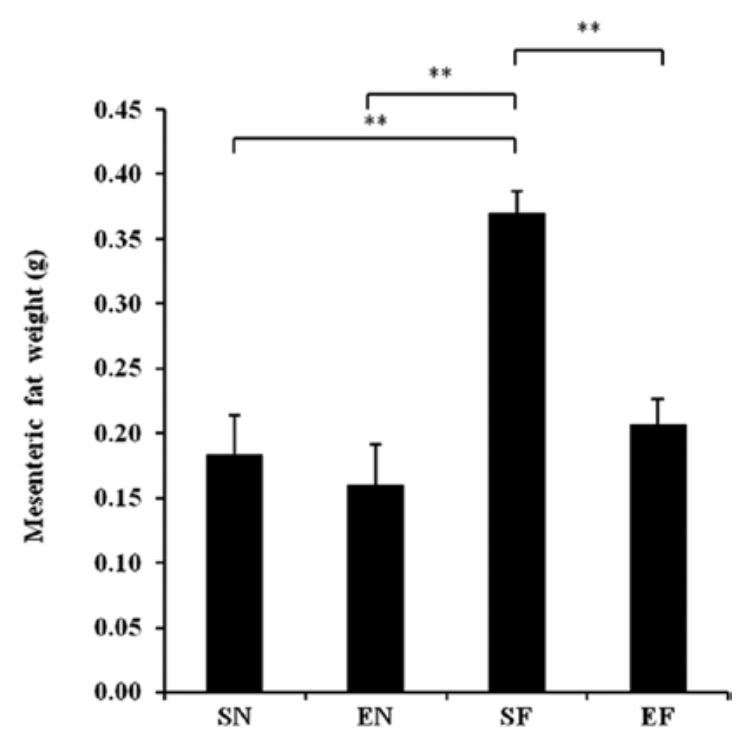

B

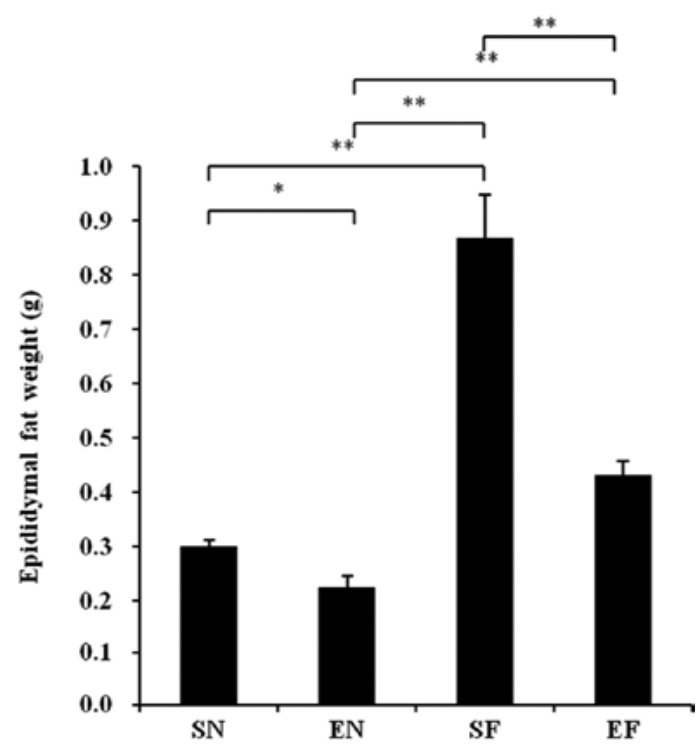

Figure 2. Effect of exercise and high-fat diet on body fat in C57BL/6J mice on (A) mesenteric fat weight and (B) epididymal fat weight. "P<0.05 and ** $\mathrm{P}<0.01$. $\mathrm{SN}$, normal diet in non-exercise groups; EN, normal diet in exercise groups; SF, high-fat diet in non-exercise groups; EF, high-fat diet in exercise groups.

(TG) concentrations were measured using the Cholesterol E-test Wako and Triglyceride E-test Wako kits (Wako Pure Chemical, Ind., Osaka, Japan), respectively. Mice plasma adiponectin (Otsuka Pharmaceutical, Co., Tokyo, Japan) and nesfatin-1 levels (Phoenix Pharmaceuticals, Inc., CA, USA) were measured by their corresponding ELISA-based kits.

Statistics. Analysis of variance followed by Bonferroni's test were used to assess the differences among the groups. A value of $\mathrm{P}<0.05$ was considered to be statistically significant.

\section{Results}

The weekly body weight in the SF group significantly increased compared to that in the other groups $(\mathrm{P}<0.01)$. The body weight in the EF group significantly increased at week $1(\mathrm{P}<0.01)$ and at weeks 3 and $4(\mathrm{P}<0.05)$ compared to that in the EN group (Fig. 1A). The motor activity did not differ significantly between the EN and EF groups, but an increasing trend was observed in the motor activity in the EF group compared to that in the EN group (Fig. 1B). The mean daily food intake (Kcal) in the high-fat diet groups significantly increased compared to that in the normal diet groups $(\mathrm{P}<0.01)$. The mean daily food intake (Kcal) in the exercise groups significantly increased compared to that in the non-exercise groups $(\mathrm{P}<0.01)$ (Fig. 1C). The temperature of the mice in the EF group significantly increased compared to that of the mice in the SN group $(\mathrm{P}<0.05)$ (Fig. 1D).

The mesenteric fat in the SF group significantly increased compared to that in the EF and the other groups $(\mathrm{P}<0.01)$, while the mesenteric fat in the EF group showed no significant difference compared to that in the normal diet groups (Fig. 2A). The epididymal fat in the SF group significantly increased compared to that in the other groups $(\mathrm{P}<0.01)$, while the epididymal fat in the EF group did not significantly differ from that in the SN group (Fig. 2B).

The glucose concentration in the SF group significantly increased compared to that in the normal diet groups $(\mathrm{P}<0.01)$, while the blood glucose level in the EF group did not differ significantly from that in the normal diet groups (Fig. 3A). The plasma insulin concentration in the SF group significantly increased compared to that in the other groups $(\mathrm{P}<0.01)$, while the plasma insulin concentration in the EF group did not differ significantly from that in the normal diet groups (Fig. 3B). The plasma TG concentration in the SF group significantly increased compared to that in the normal diet groups $(\mathrm{P}<0.01)$, while the plasma $\mathrm{TG}$ concentration in the EF group did not differ significantly from that in the normal diet groups (Fig. 3C). The plasma cholesterol concentration in the high-fat diet groups significantly increased compared to that in the normal diet groups $(\mathrm{P}<0.01)$ (Fig. 3D). With the high-fat diet, the plasma cholesterol concentration in the exercise group significantly decreased compared to that in the non-exercise group $(\mathrm{P}<0.05)$. The plasma adiponectin level in the SF group significantly decreased compared to that in the normal diet groups $(\mathrm{P}<0.05$ and $\mathrm{P}<0.01)$. The plasma adiponectin level in the EF group significantly decreased compared to that in the $\mathrm{SN}$ group $(\mathrm{P}<0.05)$ (Fig. 3E). The plasma adiponectin concentration did not differ significantly between the EF and EN groups. The plasma nesfatin-1 level in the SF group significantly decreased compared to that in the SN group $(\mathrm{P}<0.05)$, while the plasma nesfatin-1 level in the EF group did not differ significantly from that in the normal diet groups (Fig. 3F).

\section{Discussion}

To date, it has been reported that exercise is closely related to body weight, especially the accumulation of fat (17). Physical exercise is a strategy used to counteract obesity, as it lowers the energetic balance by increasing energy expenditure (18). This study reveals that exercise suppresses body weight gain caused by a high-fat diet. Although the high-fat diet increased visceral fat, visceral fat did not increase in the exercise groups. The food intake (Kcal) of the mice in the exercise 
A

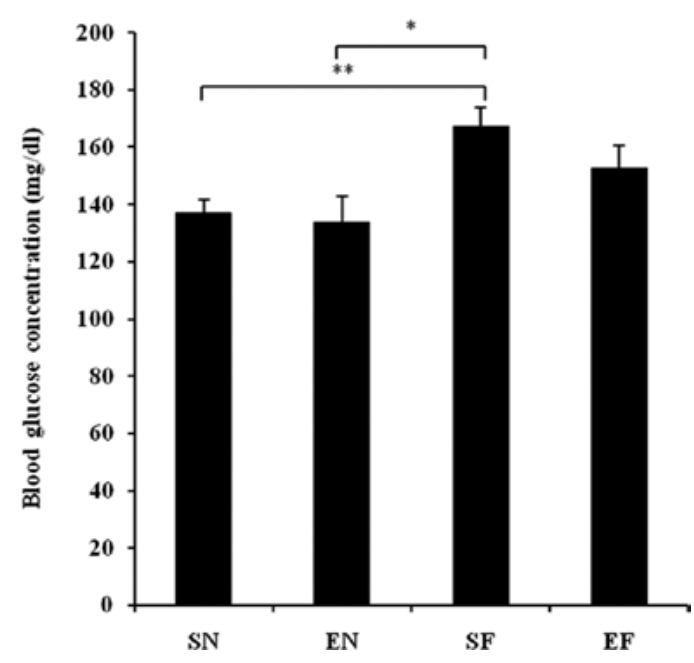

C

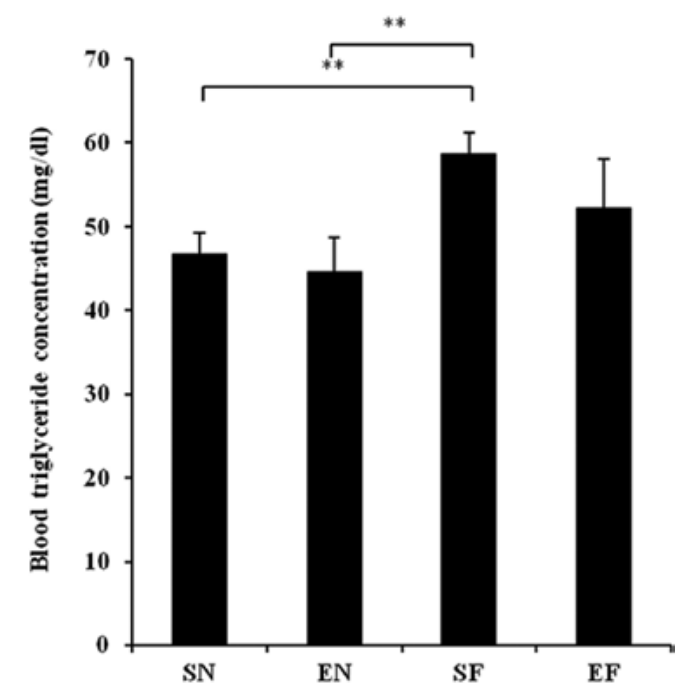

$\mathbf{E}$

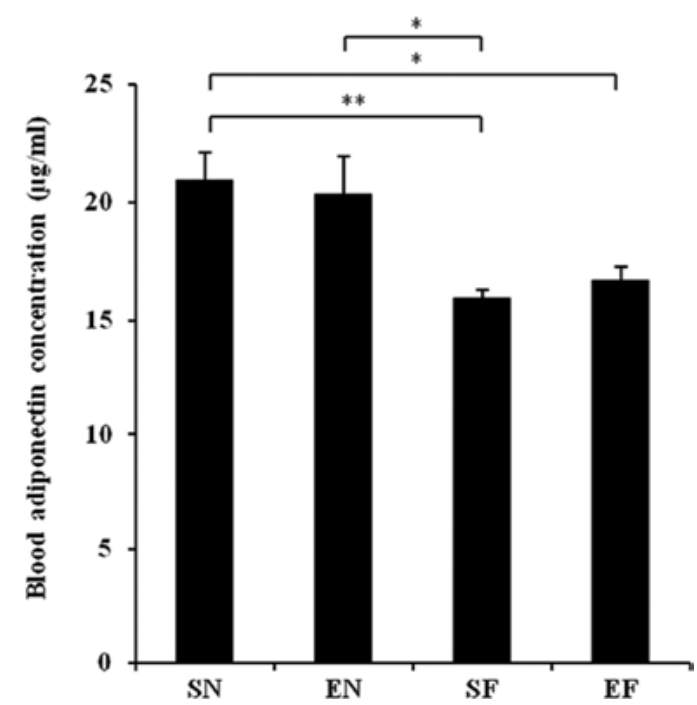

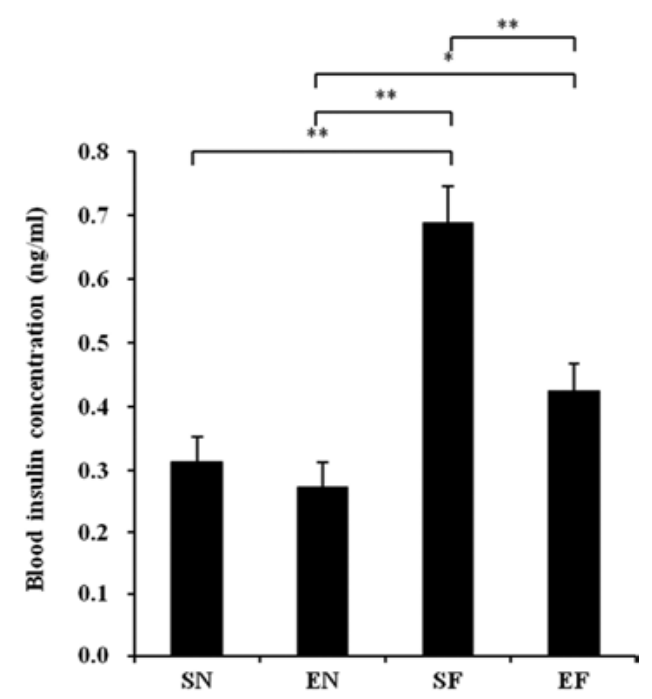

D

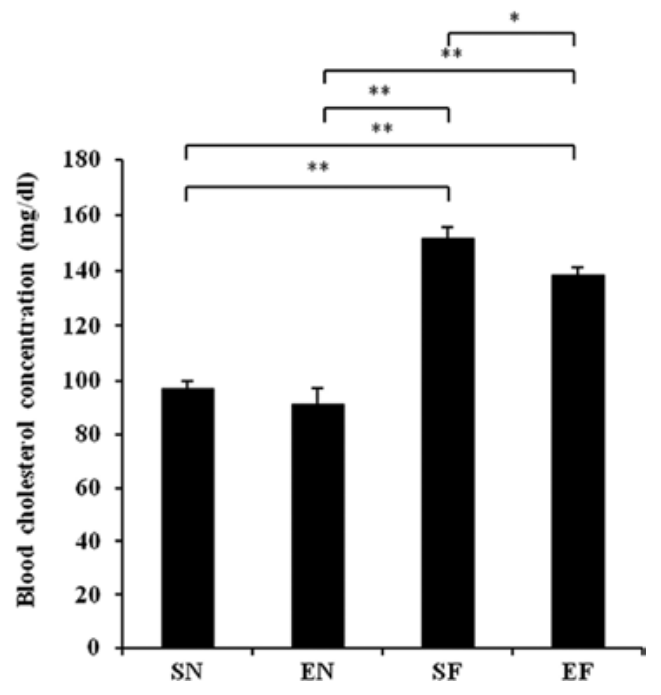

$\mathbf{F}$

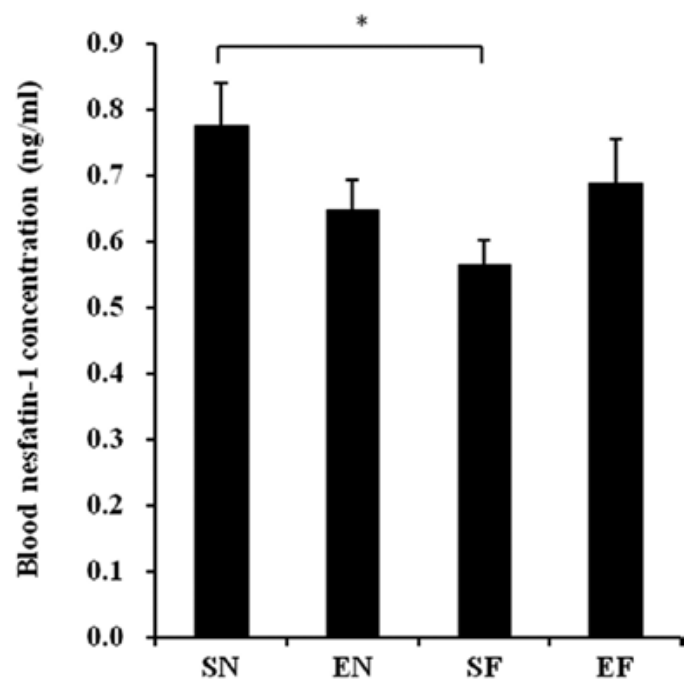

Figure 3. Effect of exercise and high-fat diet in C57BL/6J mice on (A) blood glucose concentration, (B) blood insulin concentration, (C) blood triglyceride concentration, (D) blood cholesterol concentration, (E) blood adiponectin concentration and (F) blood nesfatin-1 concentration. * $\mathrm{P}<0.05$ and ${ }^{* *} \mathrm{P}<0.01$. $\mathrm{SN}$, normal diet in non-exercise groups; EN, normal diet in exercise groups; SF, high-fat diet in non-exercise groups; EF, high-fat diet in exercise groups.

groups significantly increased compared to that in the nonexercise groups. It is thought that exercise increases the need for energy intake, resulting in an increased appetite.
It is known that the energy generated after the intake of a high-fat diet is relatively higher than that generated after a normal diet (19). In this study, the temperature of the mice 
in the EF group significantly increased compared to that of the mice in the SN group. In addition, an increasing trend was observed in the temperature of the mice in the EF group compared to that of the mice in the EN group. In the EF group, the glucose and insulin concentrations that increased with the high-fat diet, decreased. Hence, it is considered that exercise increases insulin sensitivity. In addition, in the EF group, TG and total cholesterol concentrations that increased with the high-fat diet, decreased.

It has previously been shown that adiponectin has certain effects on insulin sensitivity, the regulation of blood glucose, the repair of vascular lesions and the suppression of arteriosclerosis (20). Hattori et al have shown that adiponectin activates the AMP-activated protein kinase in the skeletal muscle and liver, and thus promotes the combustion of fatty acids and the uptake of glucose to improve insulin resistance (21). In this study, the plasma adiponectin level in the high-fat diet groups significantly decreased compared to that in the SN group. However, in the exercise groups, the plasma adiponectin level did not differ significantly between the high-fat diet and normal diet groups.

No reports are as yet available on the effects of exercise and high-fat diet on nesfatin-1. In this study, the plasma nesfatin-1 level in the SF group significantly decreased compared to that in the SN group, while exercise under the high-fat diet antagonized this significant decrease. It has recently been reported that the fasting levels of nesfatin-1 are significantly lower in type 2 diabetes mellitus patients than in healthy subjects (22). In addition, Su et al have reported that the intravenous administration of nesfatin-1 reduces the blood glucose level in hyperglycemic $\mathrm{db} / \mathrm{db}$ mice (23). Moreover, nesfatin-1-like immunoreactivity has been detected in human and rat islet $\beta$ cells (24). Therefore, it is suggested that plasma nesfatin is associated with lifestyle-related diseases, such as diabetes and obesity.

In conclusion, a high-fat diet decreases plasma adiponectin and nesfatin levels, but this decrease is suppressed by exercise. Accordingly, it has been revealed that not only diet, but also exercise, are important in the development and progression of lifestyle-related diseases.

\section{References}

1. Yamauchi T, Oike Y, Kamon J, et al: Increased insulin sensitivity despite lipodystrophy in Crebbp heterozygous mice. Nat Genet 30: 221-226, 2002

2. Hara K, Boutin P, Mori Y, et al: Genetic variation in the gene encoding adiponectin is associated with an increased risk of type 2 diabetes in the Japanese population. Diabetes 51: 536-540, 2002.

3. Okamoto Y, Arita Y, Nishida M, et al: An adipocyte-derived plasma protein, adiponectin, adheres to injured vascular walls. Horm Metab Res 32: 47-50, 2000.

4. Arita Y, Kihara S, Ouchi N, et al: Paradoxical decrease of an adipose-specific protein, adiponectin, in obesity. Biochem Biophys Res Commun 257: 79-83, 1999.
5. Hotta K, Funahashi T, Arita Y, et al: Plasma concentrations of a novel, adipose-specific protein, adiponectin, in type 2 diabetic patients. Arterioscler Thromb Vasc Biol 20: 1595-1599, 2000.

6. Hulver MW, Zheng D, Tanner CJ, et al: Adiponectin is not altered with exercise training despite enhanced insulin action. Am J Physiol Endocrinol Metab 283: E861-E865, 2002.

7. Boudou P, Sobngwi E, Mauvais-Jarvis F, Vexiau P and Gautier JF: Absence of exercise-induced variations in adiponectin levels despite decreased abdominal adiposity and improved insulin sensitivity in type 2 diabetic men. Eur J Endocrinol 149: 421-424, 2003.

8. Yatagai T, Nishida Y, Nagasaka S, et al: Relationship between exercise training-induced increase in insulin sensitivity and adiponectinemia in healthy men. Endocr J 50: 233-238, 2003.

9. Oh-I S, Shimizu H, Satoh T, et al: Identification of nesfatin-1 as a satiety molecule in the hypothalamus. Nature 443: 709-712, 2006.

10. Brailoiu GC, Dun SL, Brailoiu E, Inan S, Yang J, Chang JK and Dun NJ: Nesfatin-1: distribution and interaction with a G protein-coupled receptor in the rat brain. Endocrinology 148: 5088-5094, 2007.

11. Kohno D, Nakata M, Maejima Y, et al: Nesfatin-1 neurons in paraventricular and supraoptic nuclei of the rat hypothalamus coexpress oxytocin and vasopressin and are activated by refeeding. Endocrinology 149: 1295-1301, 2008.

12. Stengel A, Goebel M, Yakubov I, et al: Identification and characterization of nesfatin-1 immunoreactivity in endocrine cell types of the rat gastric oxyntic mucosa. Endocrinology 150: 232-238, 2009.

13. Shimizu H,Oh-I S, Hashimoto K, et al: Peripheral administration of nesfatin-1 reduces food intake in mice: the leptin-independent mechanism. Endocrinology 150: 662-671, 2009.

14. Price TO, Samson WK, Niehoff ML and Banks WA: Permeability of the blood-brain barrier to a novel satiety molecule nesfatin-1. Peptides 28: 2372-2381, 2007.

15. Cowley MA and Grove KL: To be or NUCB2, is nesfatin the answer? Cell Metab 4: 421-422, 2006.

16. Schwartz MW, Woods SC, Porte D Jr, Seeley RJ and Baskin DG: Central nervous system control of food intake. Nature 404: 661-671, 2000

17. Cox KL, Burke V, Beilin LJ and Puddey IB: A comparison of the effects of swimming and walking on body weight, fat distribution, lipids, glucose, and insulin in older women-the Sedentary Women Exercise Adherence Trial 2. Metabolism 59: 1562-1573, 2010.

18. MacLean PS, Higgins JA, Wyatt HR, et al: Regular exercise attenuates the metabolic drive to regain weight after long-term weight loss. Am J Physiol Regul Integr Comp Physiol 297: 793-802, 2009.

19. Michailidou Z, Carter RN, Marshall E, et al: Glucocorticoid receptor haploinsufficiency causes hypertension and attenuates hypothalamic-pituitaryadrenal axis and blood pressure adaptions to high-fat diet. FASEB J 22: 3896-3907, 2008.

20. Qi Y, Takahashi N, Hileman SM, et al: Adiponectin acts in the brain to decrease body weight. Nat Med 10: 524-529, 2004.

21. Hattori Y, Nakano Y, Hattori S, Tomizawa A, Inukai K and Kasai K: High molecular weight adiponectin activates AMPK and suppresses cytokine-induced NF-kappaB activation in vascular endothelial cells. FEBS Lett 582: 1719-1724, 2008.

22. Li QC, Wang HY, Chen X, Guan HZ and Jiang ZY: Fasting plasma levels of nesfatin-1 in patients with type 1 and type 2 diabetes mellitus and the nutrient-related fluctuation of nesfatin-1 level in normal humans. Regul Pept 159: 72-77, 2010.

23. Su Y, Zhang J, Tang Y, Bi F and Liu JN: The novel function of nesfatin-1: anti-hyperglycemia. Biochem Biophys Res Commun 391: 1039-1042, 2010

24. Foo KS, Brauner $\mathrm{H}$, Ostenson $\mathrm{CG}$ and Broberger C: Nucleobindin-2/nesfatin in the endocrine pancreas: distribution and relationship to glycaemic state. J Endocrinol 204: 255-263, 2010. 